Actas del Seminario Internacional Destinos Turísticos Inteligentes:

nuevos horizontes en la investigación y gestión del turismo

Universidad de Alicante, 26 y 27 de octubre de 2017

\title{
Caso de estudio: diseño e implementación del soporte tecnológico de un sistema de indicadores de turismo sostenible ${ }^{1}$
}

\author{
Rossi Jiménez, Carlos \\ Universidad de Málaga, España \\ Departamento de Lenguajes y Ciencias de la Computación \\ rossi@uma.es \\ Guevara Plaza, Antonio \\ Universidad de Málaga, España \\ Departamento de Lenguajes y Ciencias de la Computación \\ guevara@uma.es \\ Navarro-Jurado, Enrique \\ Universidad de Málaga, España \\ Departamento de Geografía \\ enavarro@uma.es \\ Caselli Fernández, Javier \\ Universidad de Málaga, España \\ Departamento de Lenguajes y Ciencias de la Computación \\ javiercaselli@uma.es \\ Perea-Medina, María Jesús \\ Universidad de Málaga, España \\ Facultad de Turismo \\ maria_vva_vva@hotmail.com
}

\section{Resumen}

Cualquier destino turístico que afronte de manera rigurosa un proceso de reconversión a un destino turístico inteligente debe dotarse de herramientas, preferiblemente automatizadas, que le permitan medir la eficacia de dicho proceso. En

1. La presente comunicación se enmarca en el Proyecto de Investigación «Nuevos enfoques para la planificación y gestión del territorio turístico: conceptualización, análisis de experiencias y problemas. Definición de modelos operativos para destinos turísticos inteligentes» (Proyecto CSO2014-59193-R) del Programa Estatal de I+D+I del Ministerio de Economía y Competitividad. 
Actas del Seminario Internacional Destinos Turísticos Inteligentes:

nuevos horizontes en la investigación y gestión del turismo

Universidad de Alicante, 26 y 27 de octubre de 2017

este trabajo se presenta una plataforma tecnológica desarrollada para el Sistema de indicadores de desarrollo turístico sostenible de Andalucía, y se analiza la viabilidad de su utilización para un Sistema de indicadores de destinos turísticos inteligentes.

Palabras clave: indicadores, cuadro de mandos, aplicación web, destino turístico inteligente, turismo sostenible

\section{Abstract}

Any tourist destination that faces a rigorous process to became a smart destination must develop a set of tools, preferably automated, that allow to measure the efficiency this process. This paper presents a technological platform developed for the Indicators System for Sustainable Tourism Development of Andalucia, and analyzes the feasibility of its use for a system of indicators to evaluate smart tourist destinations.

\section{Introducción}

Parece comúnmente aceptado que el concepto de destino turístico inteligente (DTI) requiere aún ser formalizado, lo que motiva que sea objeto de numerosos trabajos y proyectos de investigación (Boes et al., 2015), (Buhalis, Amaranggana 2013). No obstante, un análisis de la literatura permite concluir que los destinos turísticos que quieran ser etiquetados como inteligentes deberán articular su estrategia en base a diferentes áreas de actuación. Por ejemplo, en (Ivars et al., 2016), se establecen los ámbitos siguientes: gobernanza, sostenibilidad, conectividad y sensorización, sistemas de información e innovación.

Independientemente de que varíen los ámbitos en los que trabaje un destino turístico para convertirse en DTI, la eficacia de la estrategia que aplique sólo puede medirse estableciendo un conjunto adecuado de indicadores que permitan cuantificar tanto el estado del destino turístico como su evolución. Dado que el número de indicadores necesario suele ser muy elevado, y además las fuentes de información que los alimentan suelen ser muy diversas, es imprescindible dotar al destino turístico de un soporte tecnológico (Guevara, A. y Rossi, C. 2014) que optimice la gestión (consulta y administración) de dichos indicadores.

El objetivo de esta comunicación es presentar, como caso de estudio, el diseño e implementación del soporte tecnológico del sistema de indicadores de desarrollo turístico sostenible para Andalucía (en adelante SIA), en el que participan los autores de esta comunicación. Esta plataforma tecnológica, y 
Actas del Seminario Internacional Destinos Turísticos Inteligentes:

nuevos horizontes en la investigación y gestión del turismo

Universidad de Alicante, 26 y 27 de octubre de 2017

las conclusiones que se extraen de esta experiencia, constituyen, en nuestra opinión, una base de información muy útil para el desarrollo de sistemas de indicadores para destinos turísticos inteligentes.

\section{Métodos}

Los métodos aplicados en este trabajo deben ser diferenciados en dos bloques: por una parte, los propios de la definición del sistema de indicadores, y por otra, más técnica, los aplicados para el desarrollo de la plataforma tecnológica.

\subsection{Metodología del sistema de indicadores}

Antes de describir la metodología seguida para la determinación de la estructura de indicadores, es importante reseñar que esta tiene como objetivo posibilitar un doble esquema de medición (Alarcón et al., 2014):

- Debe permitir una medición estratégica, que permita cuantificar tanto el estado actual del destino turístico como el escenario al que los gestores del destino pretenden llegar.

- El sistema de indicadores debe facilitar un proceso de medición continua, de manera que, estableciendo un umbral para cada indicador, se generen alertas cuando la evolución del destino se desvíe de la estrategia marcada por los gestores. Igualmente, esta medición continua permite la detección temprana de comportamientos anómalos traducidos en términos de variaciones bruscas en la medición de algún criterio que, aun manteniéndose dentro de los umbrales establecidos, pueda ser síntoma de un riesgo evitable.

En cuanto la metodología elegida para el diseño del sistema de indicadores, es importante reseñar que se ha establecido una estructura jerárquica, con tres niveles de análisis. En el nivel más alto de la jerarquía se encuentran las denominadas áreas clave (gobernanza, territorio, vulnerabilidad, rentabilidad, diversificación, calidad e innovación), que ofrecen una visión integral del destino. Cada una de éstas áreas está compuesta por una colección de los denominados temas clave, que a su vez contienen al nivel más bajo de la jerarquía, los indicadores clave. Para dar una idea de la complejidad del trabajo realizado, es importante reseñar que el sistema definido contiene más de 300 indicadores clave, estructurado en 40 temas y 7 áreas claves. 
Actas del Seminario Internacional Destinos Turísticos Inteligentes:

nuevos horizontes en la investigación y gestión del turismo

Universidad de Alicante, 26 y 27 de octubre de 2017

En la Figura 1 se detallan las áreas y temas clave de este sistema de indicadores.

Figura 1. Jerarquía de indicadores

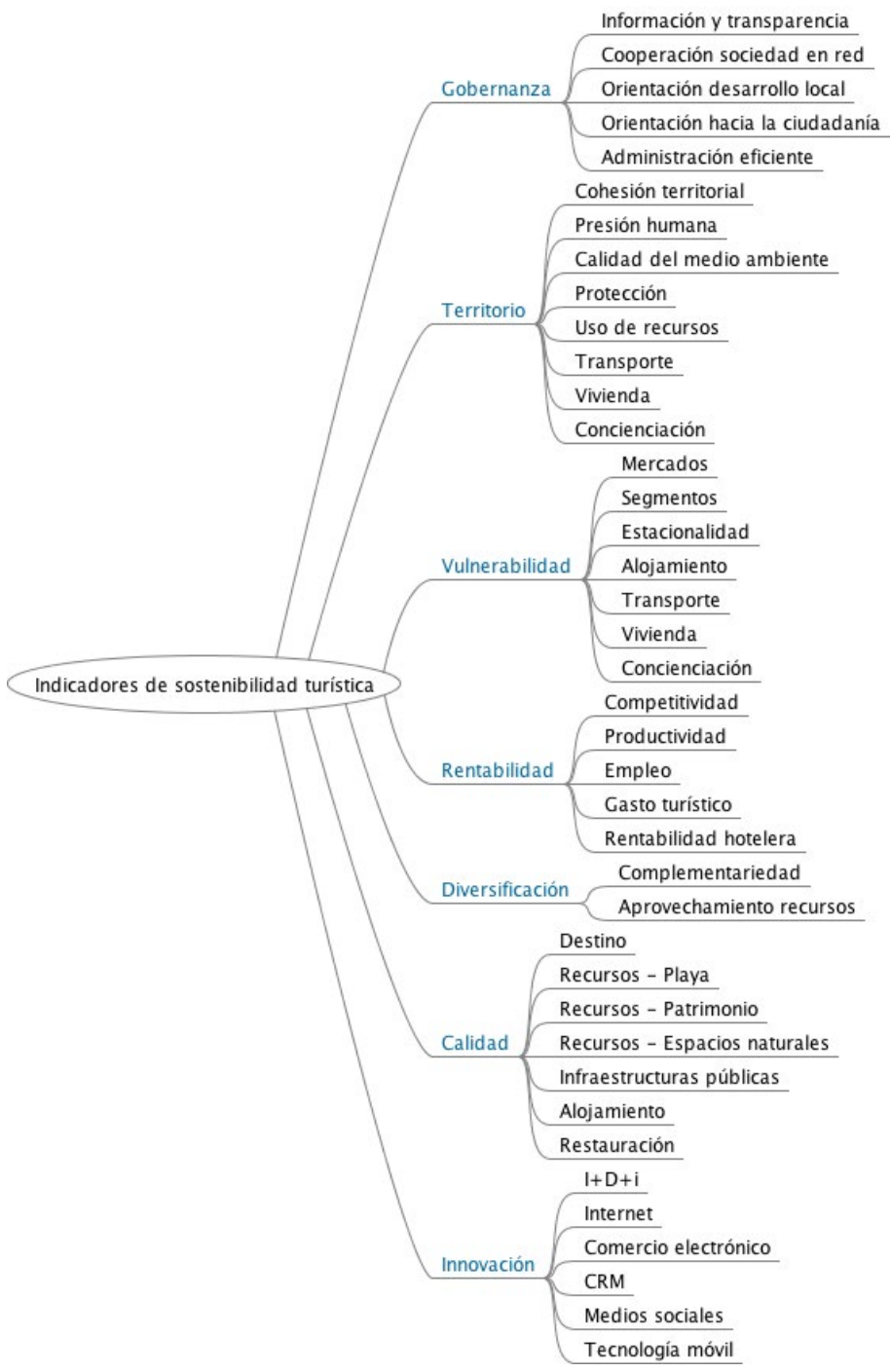

Elaboración propia 
Actas del Seminario Internacional Destinos Turísticos Inteligentes:

nuevos horizontes en la investigación y gestión del turismo

Universidad de Alicante, 26 y 27 de octubre de 2017

Para cada tema se han definido indicadores sintéticos, que permiten resumir información compleja e interdisciplinar, reduciendo el tamaño visible de la información suministrada por el conjunto de indicadores de dicho tema.

En la Figura 2 se ilustra como la aplicación desarrollada muestra al usuario los pesos e indicadores sintéticos de cada uno de los temas claves pertenecientes a un área.

Figura 2. Pesos y valores de indicadores sintéticos de cada tema clave para el área de Innovación

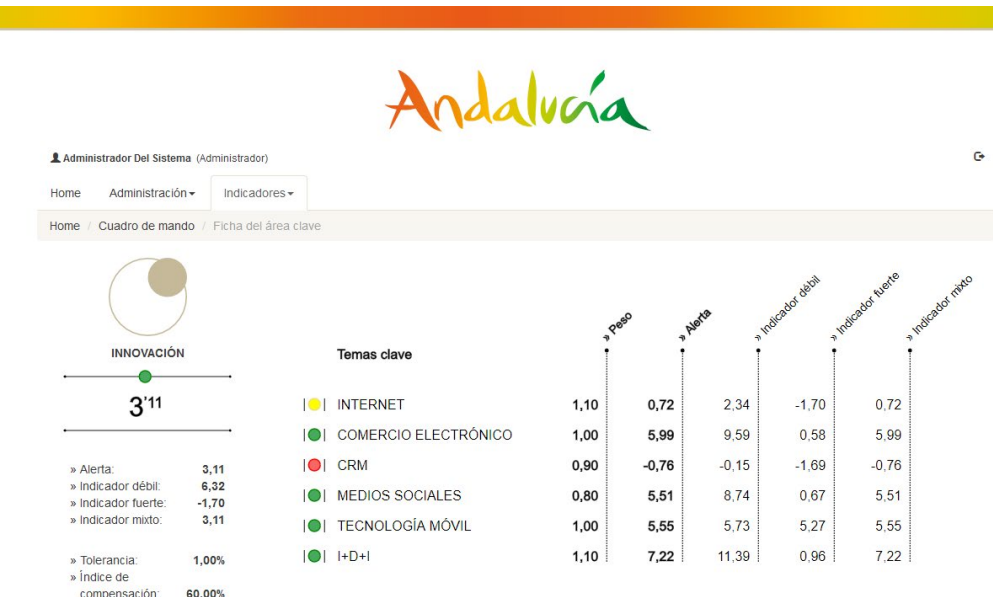

Descendiendo un nivel en la jerarquía, la aplicación muestra (véase la Figura 3) las ponderaciones y los indicadores sintéticos calculados para cada uno de los indicadores base pertenecientes a un tema clave. En la definición, cálculo y gestión de los indicadores sintéticos participan varios elementos que se describen a continuación:

- Alerta: es un modo de visualizar mediante un código de colores la variación porcentual de cada indicador. El color verde indica que el valor del indicador mejora, el amarillo que se mantiene estable y el rojo que empeora.

- Indicador débil y fuerte: en la metodología utilizada se ha propuesto la construcción de dos indicadores sintéticos para cada 
Actas del Seminario Internacional Destinos Turísticos Inteligentes: nuevos horizontes en la investigación y gestión del turismo

Universidad de Alicante, 26 y 27 de octubre de 2017

tema, siguiendo los paradigmas de sostenibilidad débil y fuerte. El paradigma de sostenibilidad débil permite la compensación entre los distintos indicadores base, y por ello el indicador débil se define como una suma ponderada de todos los indicadores base del tema. Por otro lado, el indicador fuerte no permite la compensación entre distintos indicadores, por lo que toma el valor del peor indicador (es decir, se calcula mediante el mínimo de los valores de los indicadores base), ponderado con el peso del mismo.

- Indicador mixto e índice de compensación: El indicador mixto resume de forma ponderada la información de los indicadores débil y fuerte en un único indicador. La ponderación viene dada por el índice de compensación (un valor entre 0 y 1 ), de manera que el indicador mixto es igual a la suma de producto de por el indicador débil más el producto del indicador fuerte por (1- $\lambda)$.

- Función de logro y tolerancia: la función de logro se define para medir la cercanía de los valores que toma cada indicador a su correspondiente nivel de referencia. La función de logro tiene una definición relativamente compleja, en la que juega un papel protagonista la tolerancia, que de forma simplificada se interpreta como la distancia del valor del indicador (en el año en curso) hasta el valor medio histórico, determinando los rangos de valores de la función de logro.

Para el nivel más bajo de la jerarquía, en la ficha de cada indicador base se muestran diversos detalles de éste, haciendo especial hincapié en su descripción, método de cálculo e histórico de valores (véase la Figura 4).

Los pesos asociados a los indicadores de cada tema a la hora de definir sus correspondientes indicadores sintéticos han sido establecidos por noventa expertos. A su vez los pesos asignados a cada tema dentro de las áreas claves han sido fijados por gestores políticos del gobierno regional.

Para el análisis de cada indicador, así como para la definición de los indicadores sintéticos se ha utilizado una técnica de doble punto de referencia Wierzbicki et al. (2000), Ruiz et al. (2010). Más concretamente se ha fijado para cada indicador un nivel de reserva (el valor mínimo admisible) y un nivel de aspiración (el valor deseable). 
Actas del Seminario Internacional Destinos Turísticos Inteligentes: nuevos horizontes en la investigación y gestión del turismo Universidad de Alicante, 26 y 27 de octubre de 2017

Figura 3. Ponderaciones e indicadores sintéticos para cada indicador base del tema clave «Internet»

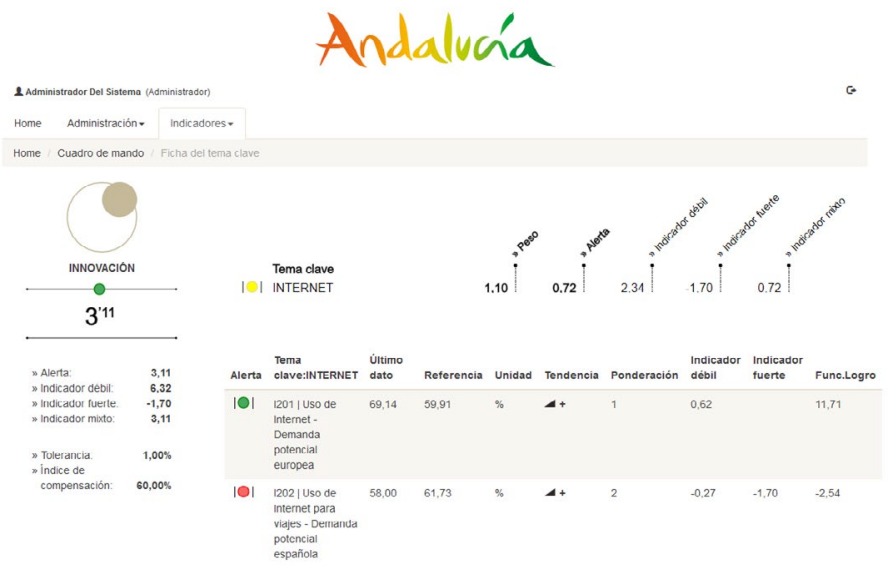

Figura 4. Indicador base «Uso de Internet para viajes - Demanda potencial Española»

\section{Andalucía}

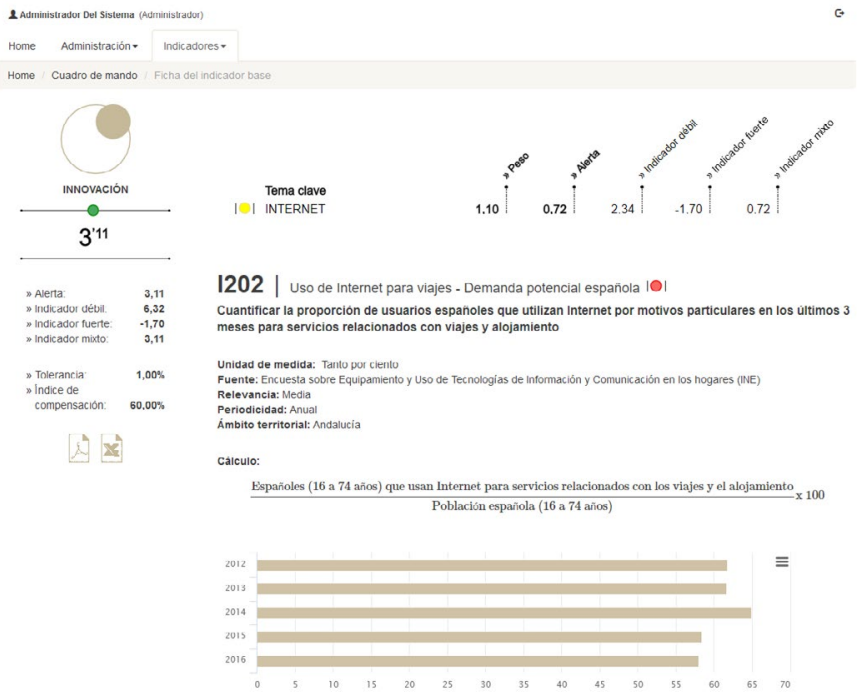


Actas del Seminario Internacional Destinos Turísticos Inteligentes:

nuevos horizontes en la investigación y gestión del turismo

Universidad de Alicante, 26 y 27 de octubre de 2017

Este extenso conjunto de indicadores permite un análisis complejo del destino turístico desde las diferentes perspectivas de la oferta, demanda, población local y administración. La amplitud del análisis hace que rebase el alcance de la gestión del destino y llegue al ámbito de otras administraciones. Como se detallará más adelante, esto añade un factor de complejidad operativa y tecnológica importante, ya que el sistema debe estar preparado para alimentarse de fuentes de datos de diversas administraciones e instituciones (del sector turístico, medioambientales, demográficas, económicas, etc.), con las consiguientes diferencias en cuanto a estructuras de datos y soporte tecnológico. Más específicamente, la información se ha extraído fundamentalmente de instituciones como Eurostat, Instituto Nacional de Estadística, Instituto de Estadística de Andalucía, Sistema de Análisis y Estadística del Turismo de Andalucía (SAETA) de la Empresa Pública para la Gestión del Turismo y del Deporte de Andalucía, Consejería de Turismo y Comercio y Consejería de Medio Ambiente, entre otros. Además de estas fuentes, también se ha obtenido información a través de encuestas o mediciones ad hoc, por ejemplo, para conocer la opinión o perspectiva de la población local.

\subsection{Metodología de desarrollo de software}

Respecto al desarrollo tecnológico de la plataforma, se opta por la aplicación de una metodología de desarrollo ágil basada en Scrum (Schwaber y Sutherland, 2016) como referencia principal. De acuerdo con esto, se determinaron diferentes sprints (concepto usado en las metodologías ágiles para referirse a las principales fases de un proyecto), cada uno de ellos actuando como contenedor de un subconjunto de requisitos funcionales. En este sentido, fieles a los principios de las metodologías ágiles, el contenido de cada sprint se fue ajustando durante el desarrollo del proyecto a los cambios sugeridos por los usuarios.

Como referencia secundaria desde el punto de vista metodológico se ha tomado Disciplined Agile Delivery (Ambler y Lines, 2012). El motivo es que esta metodología da gran importancia a la incorporación de una fase previa de concepción del proyecto, en la cual se identifican y analizan los requisitos del proyecto. De esta forma, se contrarrestan algunos de los riesgos asociados a las metodologías ágiles, motivados por la flexibilidad que imponen en la planificación de los proyectos. Este enfoque es compartido por otras metodologías como Dynamic Systems Development Method (DSDM), que 
Actas del Seminario Internacional Destinos Turísticos Inteligentes:

nuevos horizontes en la investigación y gestión del turismo

Universidad de Alicante, 26 y 27 de octubre de 2017

también incorpora una fase de pre-proyecto con la misma orientación que la fase de concepción antes citada (DSDM Consortium, 2008).

Desde el punto de vista de modelado de software, se ha utilizado UML (Booch et al. 2009) como lenguaje de representación de los diferentes modelos de casos de uso, estructura y comportamiento.

\section{Resultados}

Al igual que en el apartado anterior, diferenciaremos en los siguientes subapartados los resultados relativos a los propios sistemas de indicadores y los relativos al desarrollo de la aplicación de soporte.

\subsection{Estructura de los sistemas de indicadores}

Es conveniente recordar que el objetivo principal de este sistema de indicadores turísticos ha sido crear una información sintética y operativa para la gestión y la toma de decisiones en la Consejería de Turismo del gobierno andaluz, con el fin de controlar, evaluar resultados, determinar desviaciones y reorientar las políticas turísticas. En otras palabras, el sistema de indicadores no estaba concebido inicialmente para la evaluación de destinos turísticos inteligentes.

Por esta razón, consideramos interesante comparar el caso de estudio descrito con un marco de referencia de medición de destinos turísticos inteligentes. Para ello, se ha procedido a establecer una comparación entre el sistema de indicadores de desarrollo turístico sostenible para Andalucía con el sistema de indicadores para destinos turísticos inteligentes (en adelante SIDTI) definido en (Invat.tur, 2015).

La primera característica analizada es la estructura que cada sistema emplea para organizar y clasificar sus indicadores. En este sentido, al más alto nivel dicha clasificación en SIA se encuentran las áreas clave ya mencionadas, equivalentes a los ámbitos de SIDTI, y que se muestran en la Tabla 1. 
Actas del Seminario Internacional Destinos Turísticos Inteligentes:

nuevos horizontes en la investigación y gestión del turismo

Universidad de Alicante, 26 y 27 de octubre de 2017

Tabla 1. Nivel superior de los sistemas de indicadores

\begin{tabular}{|l|l|}
\hline \multicolumn{1}{|c|}{ SIA: Áreas Clave } & \multicolumn{1}{c|}{ SIDTI: Ámbitos } \\
\hline - Gobernanza & - Gobernanza \\
- Territorio & - Sostenibilidad / Accesibilidad \\
- Vulnerabilidad & - Conectividad / Sensorización \\
- Rentabilidad & - Sistemas de información / Inteligencia \\
- Diversificación & turística \\
- Calidad & - Innovación \\
- Innovación & - Información turística \\
& - Marketing Online \\
\hline
\end{tabular}

Se aprecia que, a nivel meramente nominativo, sólo coinciden las áreas/ ámbitos de Gobernanza e Innovación. Sin embargo, un análisis más detallado revela un cierto nivel de coincidencia entre ambos sistemas, por ejemplo entre las áreas de Territorio y Sostenibilidad y entre las áreas de Calidad y Accesibilidad (véase la Figura 5).

Un análisis más pormenorizado de todos los indicadores presentes en ambos sistemas podría revelar más paralelismos. En un trabajo futuro se pretende obtener una medida cuantitativa y cualitativa del nivel de coincidencia entre ambos sistemas.

Respecto al área de Innovación (véase la Figura 6), si bien hay innegables paralelismos, los indicadores presentes en SIDTI parecen más orientados a medir la presencia de ciertas capacidades tecnológicas (p. ej. wifi con una determinada calidad de servicio) frente a los indicadores de SIA, más orientados a medir el uso de los turistas de los recursos tecnológicos (p. ej. reservas de entradas por Internet). 
Actas del Seminario Internacional Destinos Turísticos Inteligentes: nuevos horizontes en la investigación y gestión del turismo

Universidad de Alicante, 26 y 27 de octubre de 2017

Figura 5. SIA vs SIDTI - Área de Territorio

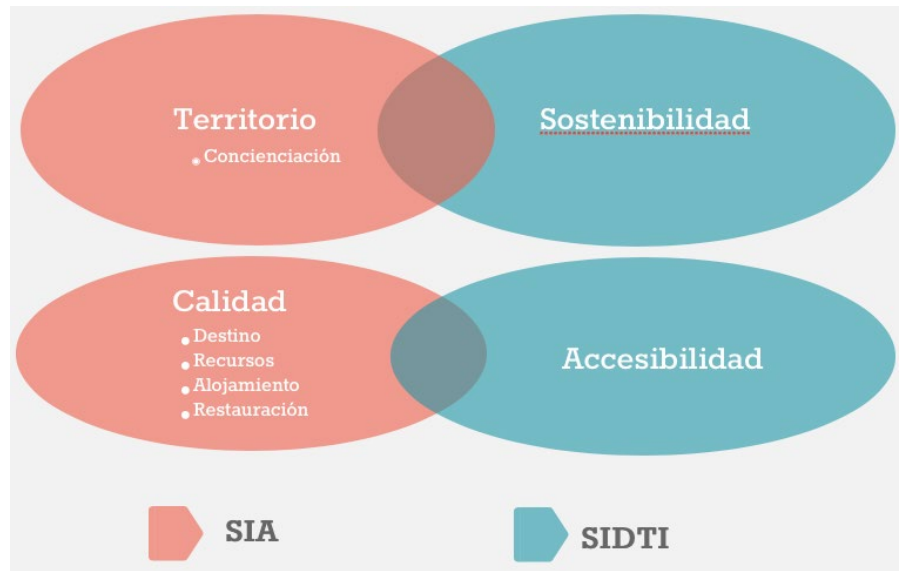

Finalmente, no se han encontrado similitudes significativas en las áreas de Vulnerabilidad, Rentabilidad y Diversificación.

Figura 6. SIA vs. SIDTI - Área de Innovación

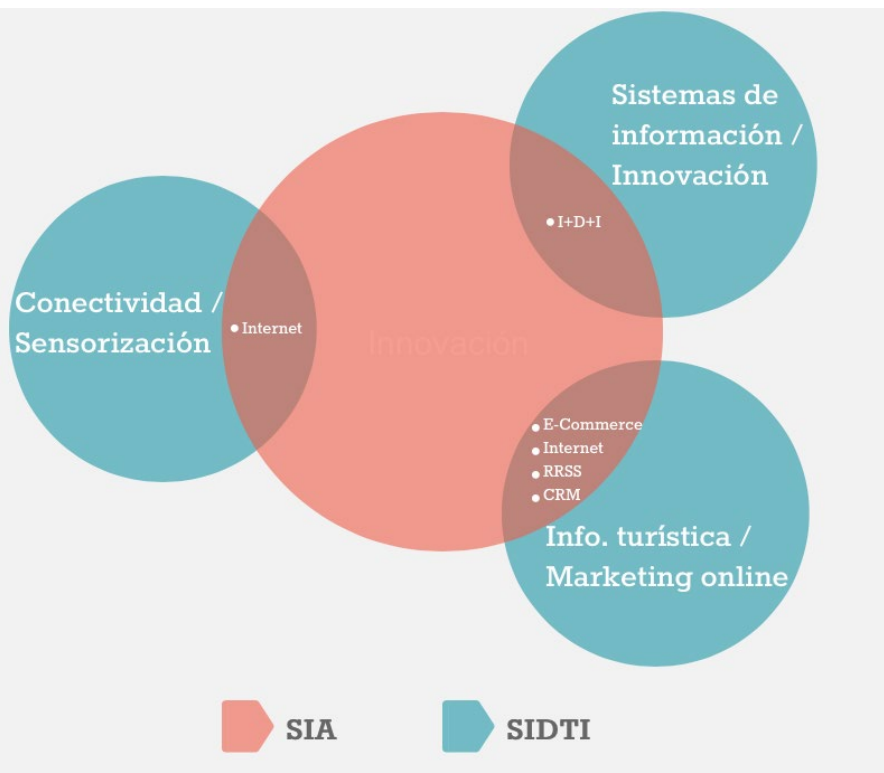


Actas del Seminario Internacional Destinos Turísticos Inteligentes:

nuevos horizontes en la investigación y gestión del turismo

Universidad de Alicante, 26 y 27 de octubre de 2017

\subsection{Aplicación de soporte del sistema de indicadores}

La aplicación desarrollada incorpora dos bloques principales desde un punto de vista funcional: por una parte, la administración y configuración de indicadores turísticos de diferentes niveles (incluyendo indicadores sintéticos), y por otra la explotación y visualización de los indicadores.

Por lo que respecta al primer bloque, los gestores del sistema de indicadores pueden definir grupos de indicadores (véase la Figura 7) y sus ponderaciones (véase la Figura 8), es decir, administrar la jerarquía ilustrada en la Figura 1. Además, para cada indicador, los gestores pueden modificar los valores de algunas de sus propiedades, como por ejemplo los umbrales o puntos de referencia (véanse la Figura 9 y la Figura 10). Otros elementos que pueden modificar en la configuración de un indicador, y probablemente los más complejos desde el punto de vista tecnológico, son los relativos a las fuentes de datos de las que se alimenta el indicador. En este sentido, desde la propia aplicación se puede modificar el patrón detallado de procesamiento de ficheros (por ejemplo .xls o.csv), dependiendo de dónde tome sus datos el indicador.

Figura 7. Administración de áreas y temas clave

\section{Andalucía}

Administración de temas y áreas clave

Parámetros generales Nuevo tema clave

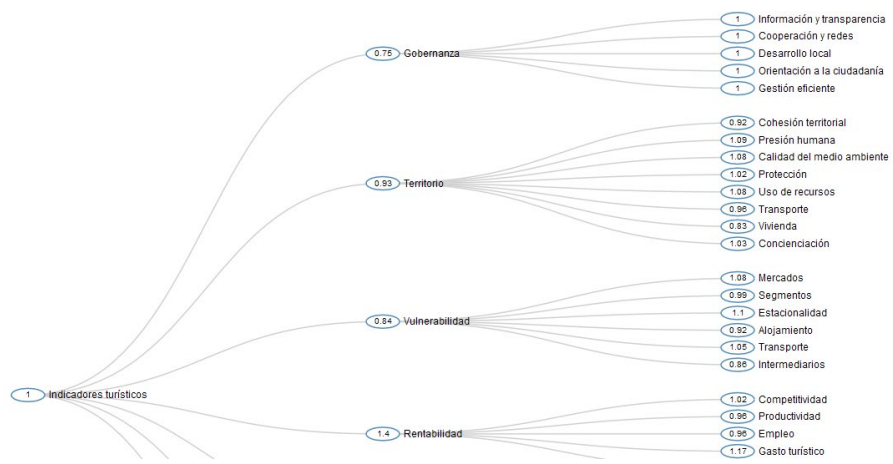


Actas del Seminario Internacional Destinos Turísticos Inteligentes:

nuevos horizontes en la investigación y gestión del turismo

Universidad de Alicante, 26 y 27 de octubre de 2017

En cuanto a las fuentes de datos, en una primera instancia se hizo un análisis de los distintos orígenes y formatos de estas, que incluían, entre otros:

- Consultas directas a bases de datos

- Cubos OLAP de distintas fuentes

- Tablas Excel

- Con datos directos obtenidos de terceros

- Con datos calculados por el personal de la empresa pública Turismo Andaluz.

Se estudió en primera instancia la viabilidad de automatizar totalmente la importación de los datos, opción que se descartó dada la complejidad de la tarea desde el punto de vista técnico. El principal inconveniente es la gran heterogeneidad de las fuentes, que imposibilitaba desarrollar métodos de importación genéricos. Además, debe tenerse en cuenta que, además de la alta complejidad, esta solución contaba con un riesgo alto debido a la que variabilidad de los formatos de origen podía hacer que el procedimiento de importación acabase fallando.

Finalmente se optó por una solución semi-automática, desarrollando un procedimiento de importación que toma como fuente una plantilla genérica de Excel en la que se pueden volcar los datos pertinentes de tantos indicadores como el usuario estime oportuno.

Sería deseable de cara al futuro que las distintas administraciones ofreciesen sus datos bajo el paradigma de datos abiertos. Además, los datos deberían publicarse de forma estructurada a través de una API estable, de forma que se facilite su consulta y tratamiento por parte de otros estamentos. 
Actas del Seminario Internacional Destinos Turísticos Inteligentes:

nuevos horizontes en la investigación y gestión del turismo

Universidad de Alicante, 26 y 27 de octubre de 2017

Figura 8. Edición de áreas clave

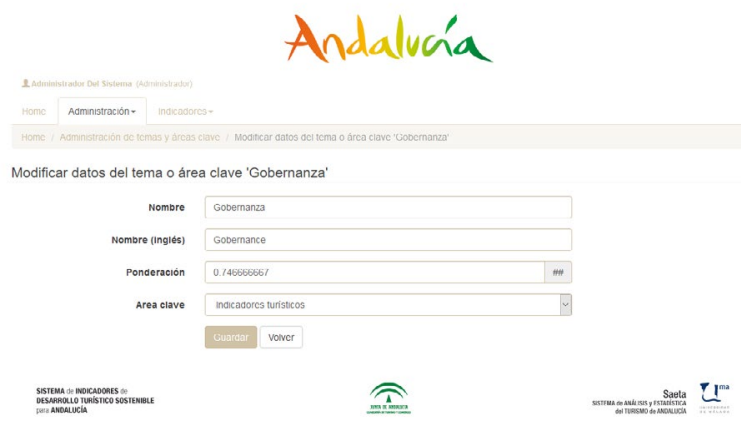

Por otro lado, evidentemente, la aplicación facilita la creación, edición y borrado de los indicadores a través de su interfaz web.

Los datos recogidos de las correspondientes fuentes se procesan para aplicar las reglas de cálculo de cada indicador. En este sentido, el sistema permite definir los parámetros comunes para el cálculo de los indicadores sintéticos de áreas, temas e indicadores base (véase la Figura 11). Además, el sistema mantiene un historial, de manera que se registran los valores de cada cálculo para analizar las sucesivas series temporales.

Figura 9. Edición de indicadores base

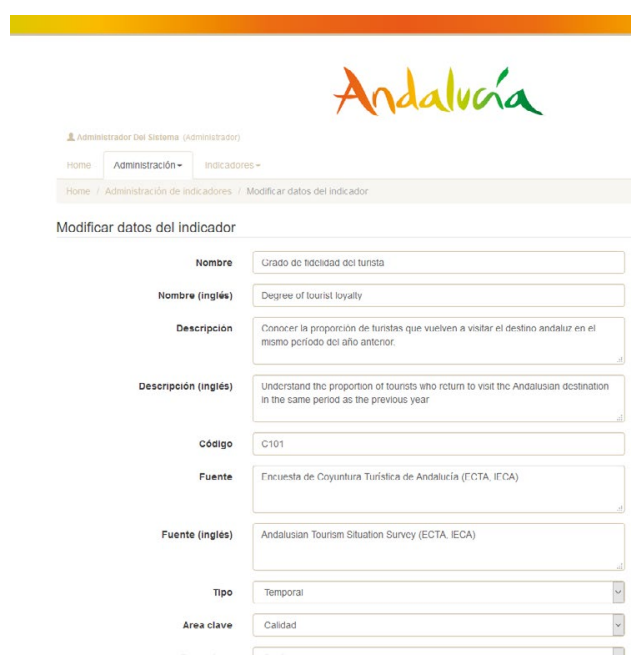


Actas del Seminario Internacional Destinos Turísticos Inteligentes:

nuevos horizontes en la investigación y gestión del turismo

Universidad de Alicante, 26 y 27 de octubre de 2017

Figura 10. Gestión de valores de indicadores base

\section{Andalucía}

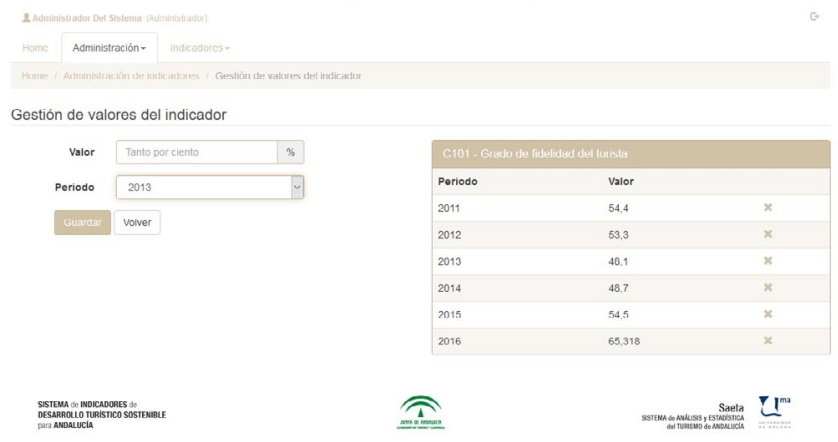

En cuanto a la explotación de la información a través de los indicadores, al desarrollar la aplicación se partió del hecho de que esta es una funcionalidad orientada fundamentalmente al soporte de la toma de decisiones por parte de los gestores del destino. Es bien conocido que un enfoque consolidado para este tipo de sistemas es el de los cuadros de mando (Eckerson, 2006). Al plantear este tipo de sistemas hay dos tareas fundamentales a abordar: la determinación de los conocidos como KPIs (key performance indicators) y desarrollar una adecuada visualización de éstos. La primera tarea queda sobradamente cubierta por la estructura del sistema de indicadores descrita en los apartados anteriores. En cuanto a la segunda, la presentación de los indicadores ha sido implementada por medio de una representación visual optimizada para la estructura jerárquica de los indicadores, mejorando así la usabilidad de la aplicación (véase la Figura 12). Cada elemento de la jerarquía tiene asociado un color que permite identificar rápidamente el estado de cada indicador, tema o área. 
Actas del Seminario Internacional Destinos Turísticos Inteligentes: nuevos horizontes en la investigación y gestión del turismo

Universidad de Alicante, 26 y 27 de octubre de 2017

Figura 11. Gestión de parámetros generales
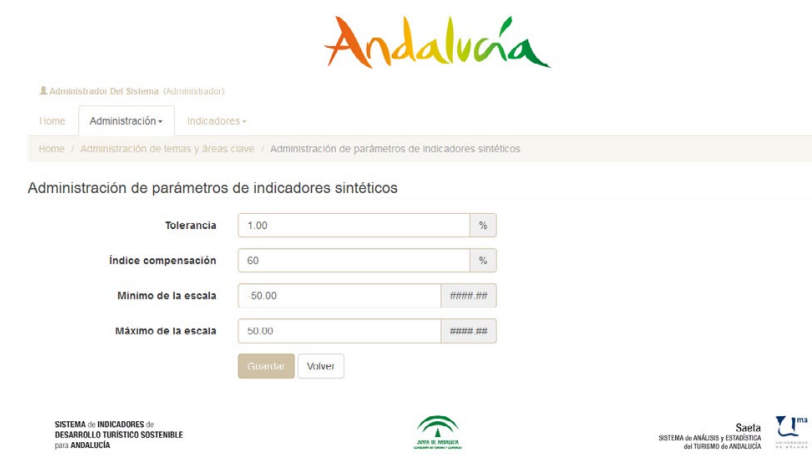

Además, el contenido del cuadro de mando se ha personalizado para cada uno de los perfiles de usuario que se han definido, restringiendo la visibilidad tanto por funcionalidades como por áreas de la organización. 
Actas del Seminario Internacional Destinos Turísticos Inteligentes:

nuevos horizontes en la investigación y gestión del turismo

Universidad de Alicante, 26 y 27 de octubre de 2017

Figura 12. Cuadro de mandos

\section{Andalucía}

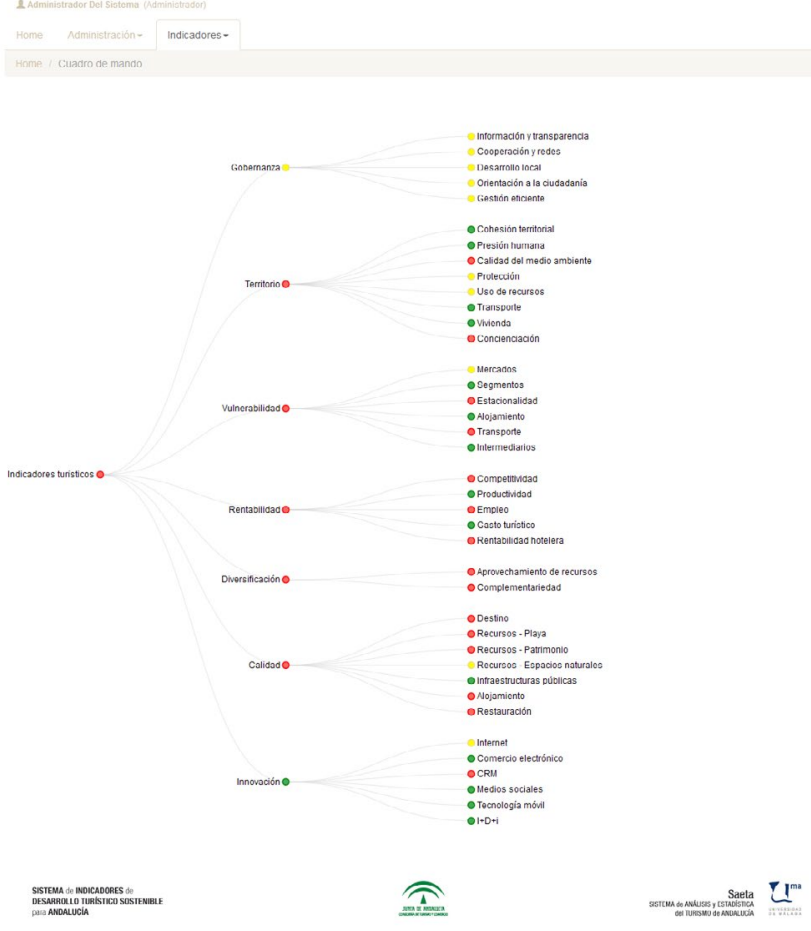

Naturalmente, el cuadro de mando permite el acceso interactivo a la información detallada de cada nivel de la jerarquía. Así, para cada indicador base se puede consultar información detallada, una gráfica con su historial y además, el estado del tema y área clave al que pertenece (véase la Figura 13).

Subiendo un nivel en la jerarquía del sistema de indicadores, la vista detallada de un tema clave muestra un resumen de todos los indicadores base que dependen de éste, así como la información propia y la de su área clave (véase la Figura 14). 
Actas del Seminario Internacional Destinos Turísticos Inteligentes:

nuevos horizontes en la investigación y gestión del turismo

Universidad de Alicante, 26 y 27 de octubre de 2017

Llegando al nivel más alto de la jerarquía, la vista detallada de un área clave muestra un resumen de todos los temas clave dependientes de esta, y a su vez una tabla con el resumen para todos los indicadores de cada uno de sus temas clave (véase la Figura 15).

Figura 13. Vista detallada de un indicador base

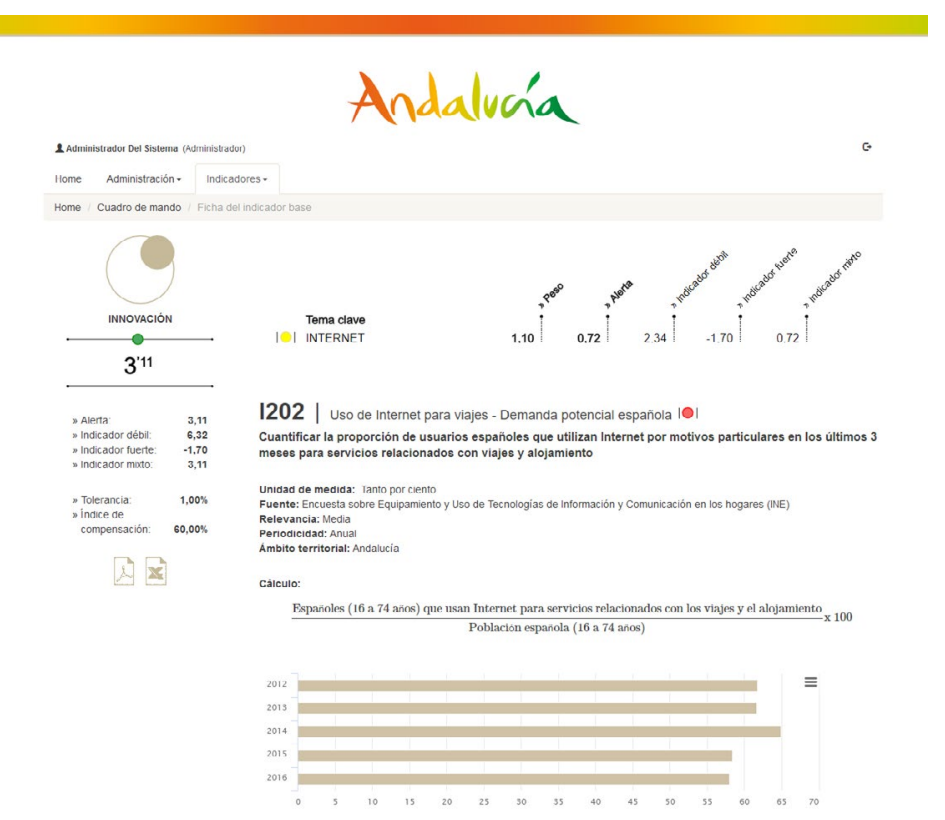


Actas del Seminario Internacional Destinos Turísticos Inteligentes: nuevos horizontes en la investigación y gestión del turismo

Universidad de Alicante, 26 y 27 de octubre de 2017

Figura 14. Vista detallada de un tema clave

\section{Andalucía}

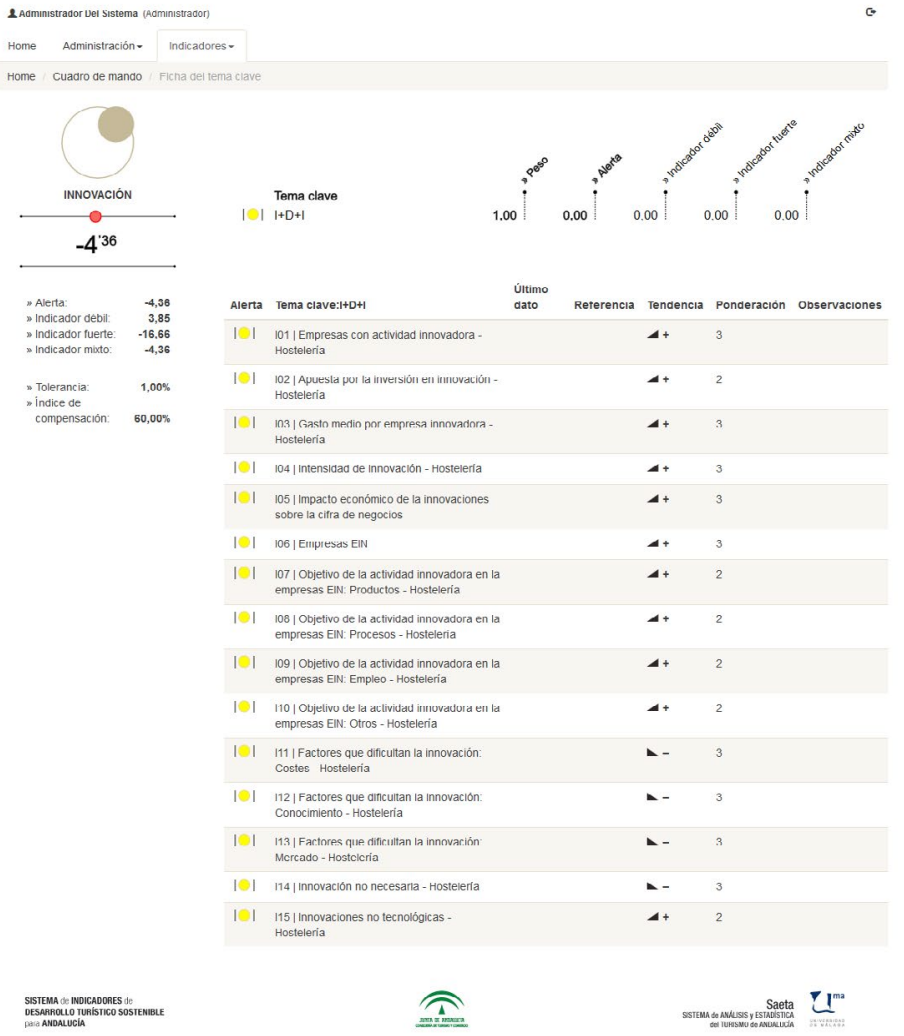


Actas del Seminario Internacional Destinos Turísticos Inteligentes: nuevos horizontes en la investigación y gestión del turismo

Universidad de Alicante, 26 y 27 de octubre de 2017

Figura 15. Vista detallada de un área clave

\section{Andalucía}

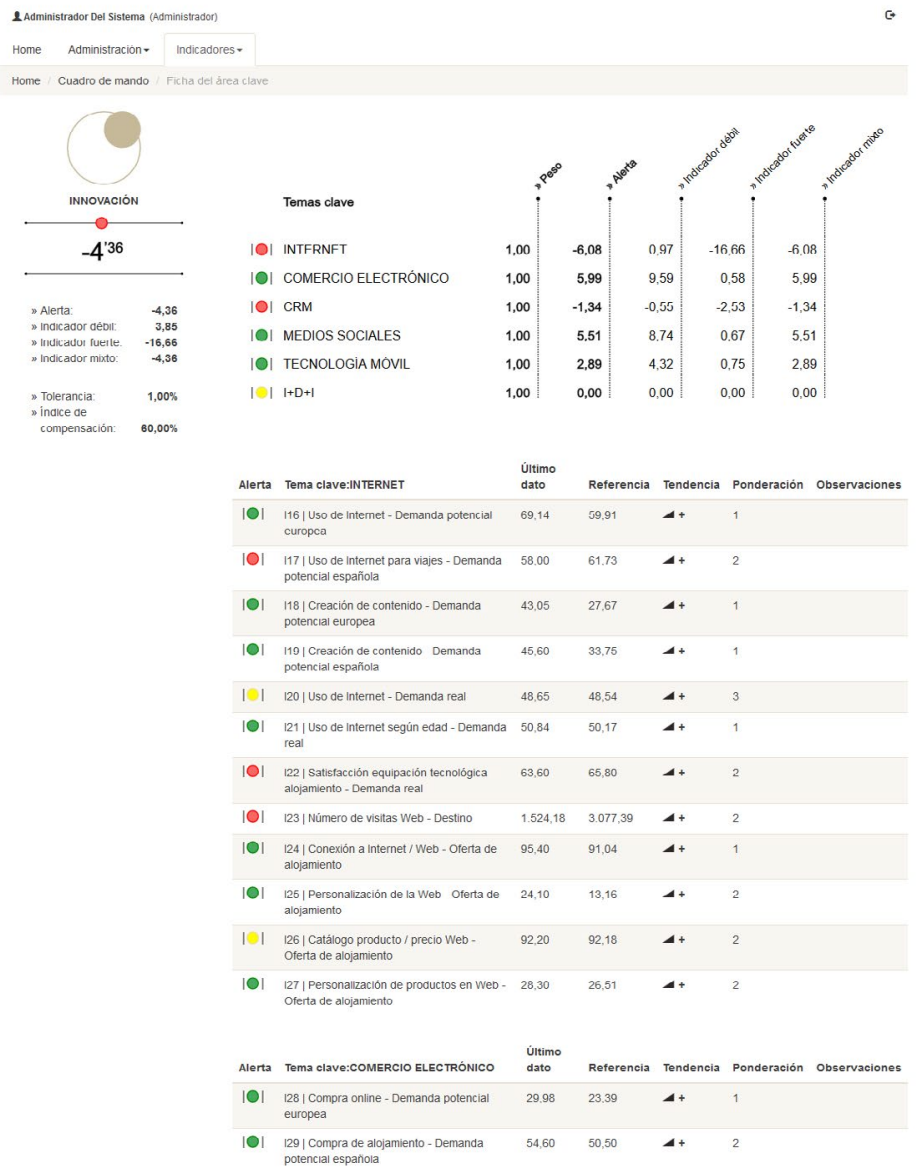


Actas del Seminario Internacional Destinos Turísticos Inteligentes: nuevos horizontes en la investigación y gestión del turismo

Universidad de Alicante, 26 y 27 de octubre de 2017

Figura 16. Arquitectura de la aplicación

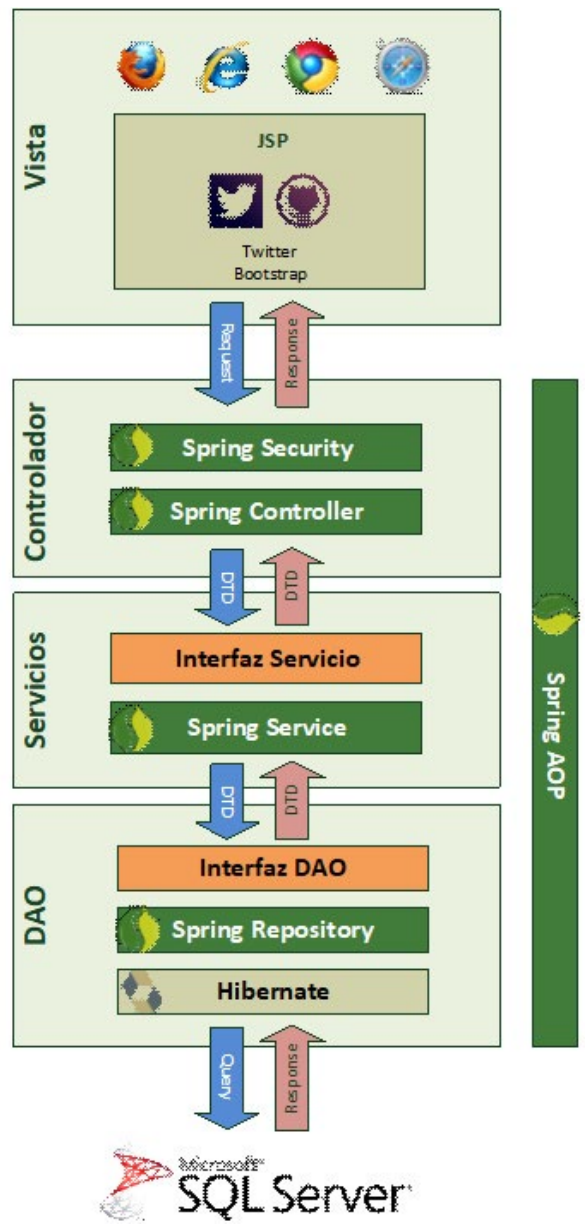

\section{Conclusiones y trabajos futuros}

Es importante resaltar varias conclusiones. Respecto al sistema de indicadores, destacar que este sistema es innovador y pionero desde su desarroIlo, no existe conocimiento de ningún organismo estadístico vinculado a un destino de ámbito regional que utilizara un sistema similar para la toma de decisiones en un destino turístico; eso convierte al sistema en un hito en el 
Actas del Seminario Internacional Destinos Turísticos Inteligentes:

nuevos horizontes en la investigación y gestión del turismo

Universidad de Alicante, 26 y 27 de octubre de 2017

modelo de información, dado que al mostrar la tendencia del destino andaluz se convierte en una herramienta de seguimiento y control de los efectos generados por la gestión y por otros factores; así mismo, está empleándose actualmente como instrumento de previsión y orientación de la política a medio/largo plazo, simulando escenarios y situaciones alternativas, lo que ayuda a disminuir el grado de incertidumbre inherente a la coyuntura turística de un destino.

En una primera aproximación, se han detectado ciertas coincidencias en la estructura del sistema de indicadores de desarrollo turístico sostenible de Andalucía y el sistema de indicadores para destinos turísticos inteligentes, a pesar de que el primero de ellos no se concibió con la orientación a Smart Destination. En una extensión de este trabajo se abordará un análisis más detallado de comparación de ambos sistemas de indicadores.

Respecto a la tecnología, aunque la plataforma tecnológica no fue desarrollada de forma explícita para evaluar destinos turísticos inteligentes, la funcionalidad implementada para la gestión y consulta de indicadores es perfectamente válida en este contexto. Además, la estructura jerárquica definida es muy adecuada para manejar sistemas con un elevado número de indicadores. Como limitación detectada en el caso de estudio, se debe destacar las dificultades encontradas en el desarrollo de la plataforma tecnológica para implementar integraciones con los sistemas externos que generan los datos de los que se alimentan los diferentes indicadores. Estas dificultades se han encontrado, por una parte a nivel operativo (dado el elevado número de instituciones generadoras de datos) y por otra parte a nivel tecnológico, por la práctica inexistencia de capas de servicios de integración en los sistemas de dichas instituciones. Este hecho hace que los datos sean suministrados mayoritariamente en forma de ficheros (usualmente en formatos csv o xls) con estructuras variables, lo que ha dificultado considerablemente la automatización de su procesado. Este inconveniente podría ser solventado de una forma relativamente sencilla si las instituciones generadoras de datos implementaran capas de servicios que suministraran feeds de datos en formato abierto.

Por otra parte, una mejora futura del sistema desarrollado sería mostrar los valores de los indicadores desagregados por municipios y no sólo para el destino turístico Andalucía. En este caso, la limitación vuelve a venir dada por algunas de las instituciones generadoras de datos, ya que suministran 
Actas del Seminario Internacional Destinos Turísticos Inteligentes:

nuevos horizontes en la investigación y gestión del turismo

Universidad de Alicante, 26 y 27 de octubre de 2017

sólo información agregada, lo que impide obtener valores de los indicadores a nivel municipal.

\section{Referencias}

Alarcón Urbistondo, P; Gallego Galán, I.; Moniche Bermejo, L; Navarro Jurado, E.; Ortega Palomo, G.; Ruiz Núñez, E. y Tineo Esteban, A.B. (2014): Sustainable Tourism Indicator System for Andalusia. 12th Global Forum on Tourism Statistics. Praga, Czech Republic (15-16 May). http://www.tsf2014prague.cz/assets/ downloads/Paper\%204.3_Ana\%20Moniche\%20Bermejo_ES.pdf

Ambler, S. y Lines, M. (2012). Disciplined Agile Delivery: A Practioner's Guide to Agile Software Delivery in the Enterprise. IBM Press

Booch, G., Rumbaugh, J. y Jacobson, I. (2009). El lenguaje Unificado de Modelado. Addison Wesley.

Buhalis, D. y Amaranggana, A. (eds.) (2013): Smart Tourism Destinations, en Xiang, Z. y Tussyadiah, I. (eds), Information and Communication Technologies in Tourism, Springer International, 553-564

Boes, K., Buhalis, D., e Inversini, A., (2015): Conceptualising Smart Tourism Destination Dimensions, en Tussyadiah, I., e Inversini, A., (eds), ENTER 2015 Proceedings, Lugano, Springer-Verlag.

DSDM Consortium (2008). DSDM Atern The Handbook. DSDM Consortium

Eckerson, W (2006). Deploying Dashboards and Scorecards. The Data Warehousing Institute.

Guevara, A. y Rossi, C. (2014). Las TICS aplicadas a la gestión de destinos turísticos. En Manual de Gestión de Destinos Turísticos. pp. 243-271. Ed. Tirant Lo Blanch. Invat.tur (2015). Destinos turísticos inteligentes. Manual operativo para la configuración de destinos turísticos inteligentes. Recuperado de http://invattur.gva. es/estudio/manual-operativo-para-la-configuracion-de-destinos-turisticos-inteligentes/

Ivars, J., Solsona, F. J., Giner, D. (2016): Gestión turística y tecnologías de la información y la comunicación (TIC): El nuevo enfoque de los destinos inteligentes, Documents d'Anàlisi Geogràfica 2016, vol. 62/2, 327-346.

Ruiz, F., Cabello, J. M., and Luque, M. (2010). An application of reference point techniques to the calculation of synthetic sustainability indicators. Journal of the Operational Research Society, 62: 189-197.

Schwaber, K. y Sutherland, J. (2016). The Scrum Guide. Recuperado de http:// www.scrumguides.org/docs/scrumguide/v2016/2016-Scrum-Guide-US.pdf\#zoom $=100$ 
Actas del Seminario Internacional Destinos Turísticos Inteligentes:

nuevos horizontes en la investigación y gestión del turismo

Universidad de Alicante, 26 y 27 de octubre de 2017

Wierzbicki, A. P., Makowski, M., and Wessels, J., editores (2000). Model-Based Decision Support Methodology with Environmental Applications. Kluwer Academic Publishers, Dordrecht. 